\title{
Renouvellement des Swissmedic Medicines Expert Committees
}

\section{Esther Wullimann}

Dr, collaboratrice scientifique et responsable de l'unité Experts, division Regulatory Operations and Development, secteur Mise sur le marché, Institut suisse des produits thérapeutiques (Swissmedic)

Lors de procédures liées aux autorisations de mise sur le marché (AMM), à la surveillance du marché ou aux autorisations d'exploitation de médicaments à usage humain ou vétérinaire, les membres des Swissmedic Medicines Expert Committees (SMEC) procèdent à un deuxième examen indépendant des documentations scientifiques.

A l'instar de toutes les autorités sanitaires majeures au plan international, Swissmedic, Institut suisse des produits thérapeutiques, recourt, pour les questions scientifiques, aux conseils d'organes d'experts externes. Il s'agit en l'occurrence des deux comités que sont le Human Medicines Expert Committee (HMEC) pour les médicaments à usage humain et le Veterinary Medicines Expert Committee (VMEC) pour les médicaments vétérinaires. La collaboration avec des experts externes est en effet essentielle pour assurer la qualité des décisions qui sont prises. L'EMA, l'Agence européenne des médicaments, dispose d'ailleurs, pour l'épauler, de plusieurs organes appelés Scientific Advisory Groups, et la FDA, l'autorité américaine de contrôle des produits thérapeutiques, travaille en étroite collaboration avec ses comités consultatifs.

\section{La collaboration avec des experts externes est en effet essentielle pour assurer la qualité des décisions qui sont prises.}

Les Swissmedic Medicines Expert Committees (SMEC) sont composés de membres ordinaires, extraordinaires et consultatifs, qui sont élus par le conseil de l'institut de Swissmedic pour une période de quatre ans. Ces spécialistes disposent de qualifications professionnelles et de connaissances pointues en médecine, en pharmacie ou en sciences naturelles ainsi que d'une longue expérience de la pratique clinique.

\section{Les experts indépendants, gages d'une assurance qualité supplémentaire}

Swissmedic recourt principalement à des experts externes dans le cadre des procédures relevant de la mise sur le marché, de la surveillance du marché et des autorisations d'exploitation dans le domaine des médicaments et des transplants standardisés, mais aussi de la surveillance du marché des dispositifs médicaux. Ses experts, qui valident les rapports d'évaluation élaborés en interne (les assessment reports), jouent un rôle important pour garantir l'objectivité des décisions scientifiques qui sont prises en entretenant un dialogue critique avec les spécialistes de Swissmedic. Le savoir-faire qu'ils ont acquis dans leur pratique clinique quotidienne est essentiel pour compléter les compétences techniques de l'institut.

Entre 2017 et 2019, les Swissmedic Medicines Expert Committees (SMEC) ont émis un total de 233 recommandations en Suisse, qui concernaient des autorisations de mise sur le marché et des utilisations de médicaments (205 pour le HMEC et 28 pour le VMEC). Leur contribution est donc indispensable pour pouvoir mettre à la disposition des médecins suisses des médicaments efficaces, sûrs et d'une qualité irréprochable pour leur pratique quotidienne.

Afin de garantir l'indépendance de ces activités de conseil, le conseil de l'institut de Swissmedic a édicté un «Code relatif à la gestion des conflits d'intérêts appli-

Tableau 1: Intégration du Human Medicines Expert Committee (HMEC) dans les activités d'examen de Swissmedic entre 2017 et 2019.

\begin{tabular}{lllll}
\hline Activité HMEC & $\mathbf{2 0 1 7}$ & $\mathbf{2 0 1 8}$ & $\mathbf{2 0 1 9}$ & Total \\
\hline Nombre de recommandations d'approbation/de rejet & 73 & 71 & 61 & 205 \\
\hline Nombre d'expertises individuelles & 24 & 33 & 23 & 80 \\
\hline Nombre d'évaluations complètes de parties de dossiers & 14 & 13 & 14 & 41 \\
\hline Total & $\mathbf{1 1 1}$ & $\mathbf{1 1 7}$ & $\mathbf{9 8}$ & $\mathbf{3 2 6}$
\end{tabular}




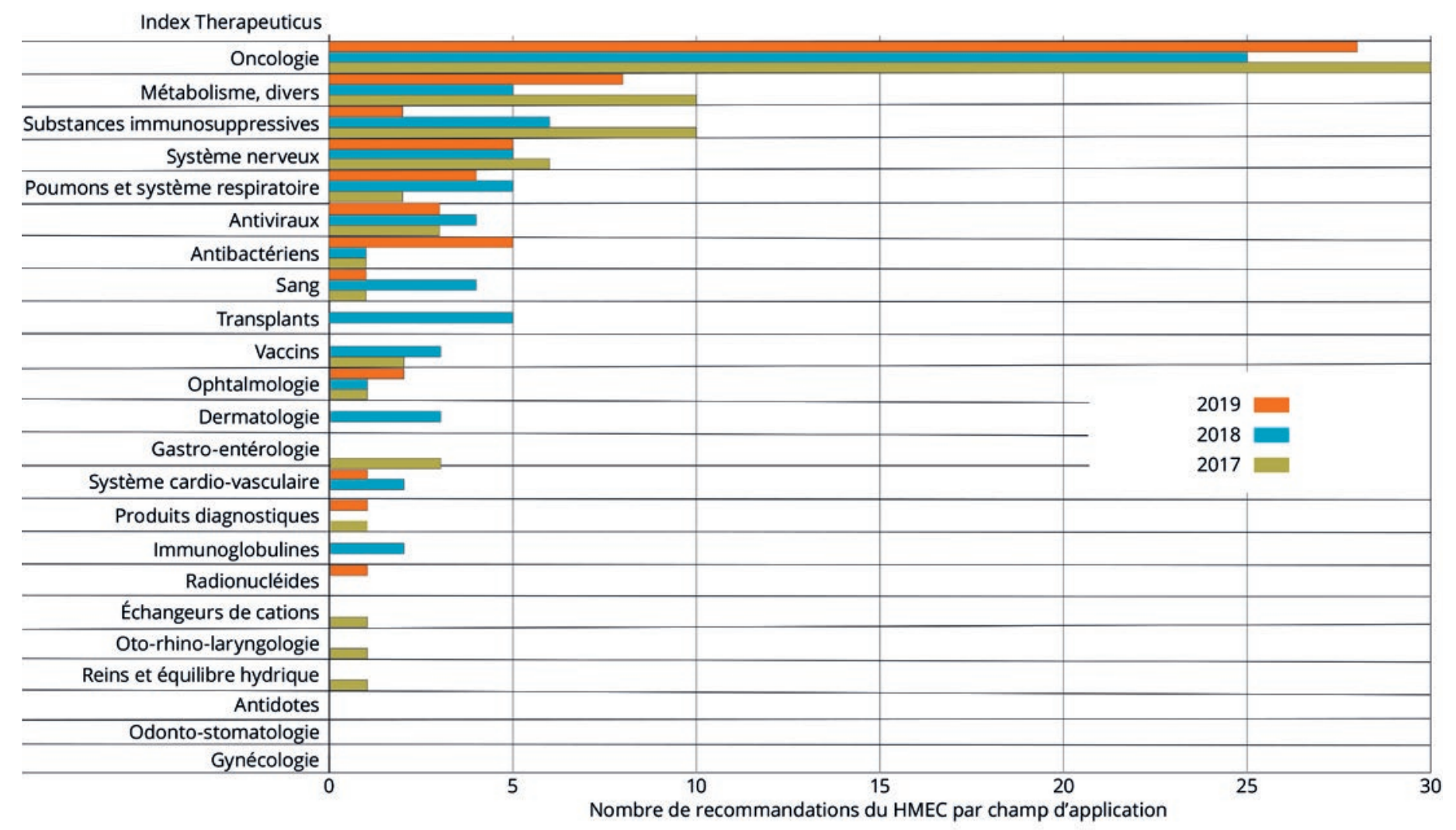

Tableau 2: Nombre de recommandations du HMEC par champ d'application entre 2017 et 2019.

cable aux Swissmedic Medicines Expert Committees», qui a principalement pour but de fournir une définition des conflits d'intérêts et d'énoncer les obligations de déclarer et les règles régissant la récusation. Tous les membres des SMEC déclarent ainsi tous les ans les intérêts qui les lient et Swissmedic les publie ensuite sur son site web. Lorsqu'un conflit d'intérêt est identifié, l'expert concerné est tenu de se récuser.

\section{Le Human Medicines Expert Committee (HMEC)}

Le HMEC est composé d'une soixantaine de membres. L'organe constitué des membres ordinaires spécialisés dans les secteurs de la médecine interne, de la pharmacologie (clinique), de la toxicologie, de l'oncologie, de la neurologie et de l'infectiologie bénéficie du soutien d'un groupe d'experts extraordinaires et consultatifs, ce qui permet de couvrir les domaines de spécialité les plus divers. Le HMEC fait ainsi appel non seulement à des spécialistes des disciplines médicales que sont la cardiologie, l'ophtalmologie ou la gastro-entérologie, mais aussi à des experts de la statistique, des phytomédicaments ou de la toxicologie réglementaire, qui viennent compléter son large éventail de compétences.
L'organe ordinaire, composé de neuf membres, se réunit tous les mois dans les locaux de Swissmedic à Berne. Il a pour mission d'évaluer l'efficacité et la sécurité de nouveaux médicaments à usage humain innovants faisant l'objet de demandes d'AMM ainsi que de demandes d'extension d'indications. La présentation des résultats de cette expertise est suivie d'une discussion en séance plénière au cours de laquelle les bénéfices et les risques sont soupesés, et qui donne lieu à une recommandation d'approbation ou de rejet des demandes déposées à l'intention de Swissmedic. Lorsque l'évaluation est sujette à controverse, la recommandation est soumise au vote. Il s'est avéré ces dernières années qu'il y avait dans la plupart des cas concordance entre l'évaluation de Swissmedic et la recommandation du HMEC. Il n'en reste pas moins que la décision finale est toujours du ressort de Swissmedic.

L'organe ordinaire, composé de neuf membres, se réunit tous les mois dans les locaux de Swissmedic à Berne.

Le HMEC a donc principalement pour tâche d'élaborer des recommandations d'approbation ou de rejet de demandes concernant de nouveaux principes actif et des extensions d'indication. Des experts extraordi- 
naires mais aussi consultatifs sont également consultés ponctuellement sur des problématiques spécifiques qui se présentent lors de procédures d'examen internes de Swissmedic (expertises individuelles). Des experts du HMEC peuvent également se charger au besoin de l'évaluation pleine et entière de parties de dossiers de demande d'AMM. Selon la spécialité de l'expert concerné, il peut alors s'agir de la documentation préclinique, clinique ou de qualité d'un médicament.

Les dossiers de demande d'AMM traités couvrent un large éventail de champs d'application. On observe cependant ces dernières années une tendance à l'accroissement du nombre de dossiers relatifs à des médicaments oncologiques (traitements immuno-oncologiques compris). Sur les 205 recommandations au total, 83 concernaient en effet ce groupe de médicaments, ce qui représente une part de $40 \%$. On peut supposer à l'heure actuelle que cette tendance va se poursuivre. Leur mandat de quatre ans s'achevant fin 2020, les SMEC devront être entièrement renouvelés en vue de la nouvelle période qui s'annonce (2021-2024). Swissmedic souhaite profiter de cette occasion pour renforcer davantage encore les compétences spécialisées de ses deux comités. L'institut recherche en particulier, en médecine humaine, des experts dans les spécialités suivantes: oncologie, pneumologie, gastro-entérologie, ophtalmologie, gynécologie, urologie, pédiatrie, allergologie, immunologie/vaccinologie, infectiologie/virologie, endocrinologie et pharmacologie clinique.

\section{L'essentiel en bref}

- Issus du monde universitaire ou professionnels de la pratique clinique, les membres des organes externes d'experts de Swissmedic ont vocation à aider l'institut dans ses tâches d'expertise.

- Leur contribution est essentielle pour garantir que les médecins suisses disposent de médicaments efficaces, sûrs et d'une qualité irréprochable dans leur pratique quotidienne.

- Entre 2017 et 2019, les experts de Swissmedic ont émis un total de 233 recommandations en Suisse, qui portaient sur des demandes de nouvelles autorisations de mise sur le marché et d'extension d'indications de médicaments.

- Pour ce qui est des médicaments à usage humain, ces recommandations avaient trait, pour une grande part, à des oncologiques.

- Swissmedic recherche des spécialistes souhaitant se porter candidats pour remplacer les membres de ses comités d'experts qui seront entièrement renouvelés fin 2020.

\section{Unterhaltung und Spannung aus der Feder von Ärzten}
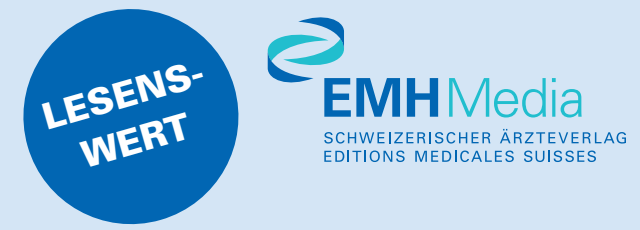

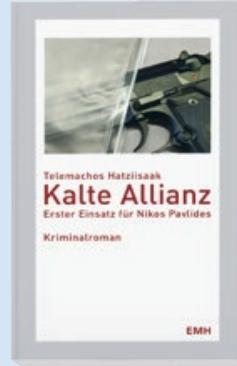

Kalte Allianz von Telemachos Hatziisaak

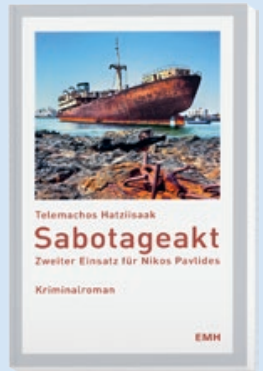

Sabotageakt von Telemachos Hatziisaak

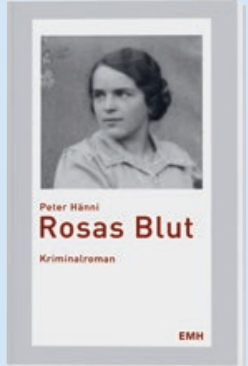

Rosas Blut von Peter Hänni

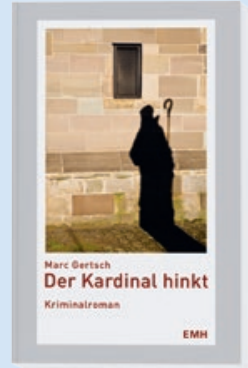

Der Kardinal hinkt von Marc Gertsch SCHWEIZERISCHER ÄRZTEVERLAG
EDITIONS MEDICALES SUISSES
Bestellung

(Fax 0614678576$)$

Ex. Kalte Allianz | Telemachos Hatziisaak | CHF 23.-

Ex. Sabotageakt | Telemachos Hatziisaak | CHF 23.-

Ex. Rosas Blut | Peter Hänni | CHF 21.50

Ex. Der Kardinal hinkt | Marc Gertsch | CHF 23.

Vorname / Name
Strasse / Nr.
PLZ / Ort
E-Mail
Datum

EMH Schweizerischer Ärzteverlag AG | Farnsburgerstrasse 8 | 4132 Muttenz auslieferung@emh.ch | shop.emh.ch 